\title{
THE USE OF EXISTING GLOBAL ELEVATION DATASET FOR ABSOLUTE ORIENTATION OF HIGH RESOLUTION IMAGE WITHOUT GCPS
}

\author{
J. Jeong, T. Kim * \\ Department of Geoinformatic Engineering, Inha University, 253 Yonghyun-Dong, Namgu, Incheon Korea \\ jaehoon@inha.edu, tezid@inha.ac.kr
}

KEY WORDS: DEM matching, global elevation data, High resolution satellite image, Absolute orientation, Sensor model

\begin{abstract}
:
For precise mapping using high resolution satellite images, various ways to eliminate the need of ground control points have been proposed. DEM matching, a technique to match two different surfaces by adjusting their position and attitude, is also one way of them. Our previous study demonstrated that DEM matching can be used for bias compensation of rigorous pushbroom sensor models. In this paper, we exploit the DEM matching technique further using existing global elevation dataset for absolute orientation of high resolution satellite image without ground control points. In particular, we investigated how the existing global DEM available worldwide can substitute ground control points when DEM matching is applied to linear pushbroom imagery. We used Spot-5 HRG with $2.5 \mathrm{~m}$ ground sampling distance(GSD) as high resolution satellite images and used SRTM-derived DEMs(DTEDs) with gird spacing of $30 \mathrm{~m}$ and $90 \mathrm{~m}$ as existing elevation dataset. We firstly established relative orientation and generated a relative DEM without use of ground control points. DEM matching was then applied between the relative DEM and the DTED. We applied DEM matching based on linear pushbroom images. Through DEM matching, accuracy of about 2 GSD or better could be achieved with in the horizontal and in the vertical direction, respectively. We confirmed automatic DEM matching processing can be accomplished for absolute orientation of pushbroom image and as results, absolute DEM can be achieved from relative DEM. We tested the applicability of 90m existing global dataset for DEM matching. Our experimental results showed the potential of existing DEM for precise mapping without ground control points. This is very encouraging in terms of applicability of the existing global elevation data. This paper will contribute to understanding of DEM matching for linear pushbroom images and of solution to precise mapping without ground control points.
\end{abstract}

\section{INTRODUCTION}

Precise mapping is one of the fundamental requirements for satellite images. As satellite sensors now provide very high resolution comparable to aerial images, more active are investigations on achieving high precision geometric information from satellite images. However, due to insufficient accuracy of the platform's ephemeris and attitude information, a large scale mapping still requires ground control points(GCPs). To avoid time-consuming and expensive GCPs measurements, various ways to eliminate the need of GCPs have been proposed.

Investigation to fully employ existing control points was implemented for automated generation of control points for new satellite images(Kim and Im, 2003). Previously ortho-rectified satellite images and height information from a suitable existing elevation dataset was used to replace the need of GCPs(Jacobsen, 2005) and similar approaches have been carried out by others(Muller et al., 2007; Gianinetto and Scaioni, 2008). More recently, the use of radar data was investigated to solve the necessary improvements of geometric accuracy of optical data such as IKONOS and ALOS-PRISM(Reinartz et al., 2011). We try here to examine DEM matching using existing global elevation data. If the existing global elevation data can replace GCPs through DEM matching, our proposal could be an efficient solution to the precise mapping using satellite image without GCPs.

DEM matching is a technique to match two different surfaces by adjusting their position and attitude. It was originally proposed for absolute orientation of perspective images(Rosenholm and Torlegard, 1988; Ebner and Strunz,
1988) and has been exploited further through several investigations(Ebner and Ohlhof, 1994; Sim and Park, 2002; Gruen and Akca, 2005). DEM matching for pushbroom image was also proposed(Ebner et al., 1991). In our previous investigation, we validated DEM matching could be used for bias compensation of pushbroom satellite images(Kim and Jeong, 2011). We applied DEM matching for linear pushbroom image with rigorous sensor models and showed that absolute orientation of pushbroom image was established from the DEM matching.

This paper attempts to exploit DEM matching technique further by using automated grid point selection and by using different elevation dataset to compare the results of absolute orientation. In our previous study, we selected grid points for DEM matching manually and removed outliers based on visual inspection. We used elevation dataset at 30 spacing for DEM matching. In this paper, we will check whether DEM matching can be fully automated including grid point selection. We will also use the publically available elevation dataset(DTED at $90 \mathrm{~m}$ spacing) and check whether this dataset can be used for precise mapping without GCPs. Firstly, we will briefly review our previous study and then discuss how to improve the previous study here.

\section{ABSOLUTE ORIENTATION OF PUSHBROOM IMAGES FROM DEM MATCHING}

In previous investigation, we examined DEM matching for absolute orientation of pushbroom images without GCPs. We

\footnotetext{
* Corresponding author: T. Kim (tezid@inha.ac.kr)
} 
firstly established relative orientation and generated a relative DEM without use of GCPs. We then applied DEM matching between the relative DEM and a reference DEM. We assumed that a ground point $(x, y, z)$ on the relative frame can be matched to the true ground point on the absolute frame $(X, Y, Z)$ by adjusting their position and attitude and that the transformation equation can be generalized as the following equation(1), where $s$ is a scale factor, $\mathbf{R}$ is a rotation matrix and $(\Delta X, \Delta Y, \Delta Z)$ are shift vector between the two DEMs. This equation is different from that of perspective images in that the rotation and shift parameters are not constant(Kim and Jeong, 2011). From DEM matching, we estimated the parameters for the equation.

$$
\left(\begin{array}{l}
X \\
Y \\
Z
\end{array}\right)=s \mathbf{R}(t)\left(\begin{array}{l}
x \\
y \\
z
\end{array}\right)+\left(\begin{array}{c}
\Delta X(t) \\
\Delta Y(t) \\
\Delta Z(t)
\end{array}\right)
$$

In our previous study, SPOT-5 stereo image at a resolution of 2.5 meter and the reference DEM at a resolution of 30 meter were employed for experiments. Relative orientation was established from SPOT-5 stereo images with only the image tiepoints and the DEM grid points(Kim and Jeong, 2010). A relative DEM at $10 \mathrm{~m}$ resolution was generated based on the relative orientation. DEM matching was applied between the relative DEM and the reference DEM and we could show the DEM matching can be used for bias compensation of linear pushbroom images without ground control points.

Here, we improve upon the previous study in two aspects. Firstly, we test an accuracy of the DEM matching with different number of grid points and perform tests using automatically extracted gird points. We will check whether automatic DEM matching can be accomplished for absolute orientation of pushbroom images without manual extraction and visual checking of grid points. Secondly, we extend our experiments to the publically available DTED at grid spacing of $90 \mathrm{~m}$ for DEM matching. We validated the feasibility of this dataset for DEM matching.

\section{DATASET USED}

For experiments, we used SPOT-5 HRG with $2.5 \mathrm{~m}$ resolution as high resolution satellite images and used two reference DEMs, one at $30 \mathrm{~m}$ grid spacing and DTED at $90 \mathrm{~m}$ grid spacing. Figure 1 presents SPOT-5 stereo pair over Daejeon, Korea used and Table 1 presents their properties, respectively. Figure 2 shows two reference DEMs over Daejeon area. GCPs for the each image were obtained by differential GPS measurements and they were used only to assess the accuracy of absolute orientation through DEM matching.
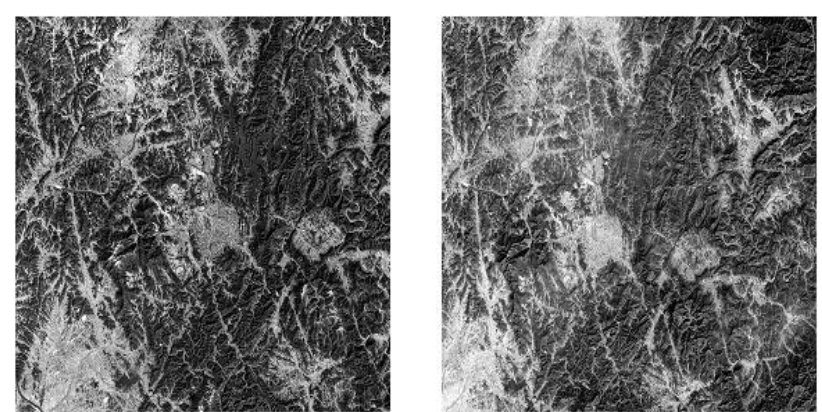

Figure 1. SPOT-5 stereo pair over Daejeon, Korea

Table 1. The properties of the SPOT-5 stereo pair used.

\begin{tabular}{|c|c|c|}
\hline ID & Left & Right \\
\hline Sensor & SPOT-5 & SPOT-5 \\
\hline Spatial Resolution & $2.5 \mathrm{~m}$ & $2.5 \mathrm{~m}$ \\
\hline Date of Acquisition & 20 Dec 2003 & 11 Dec 2003 \\
\hline Tilt angle & $18.49^{\circ}$ & $-25.53^{\circ}$ \\
\hline No. of GCPs & 18 & 18 \\
\hline
\end{tabular}
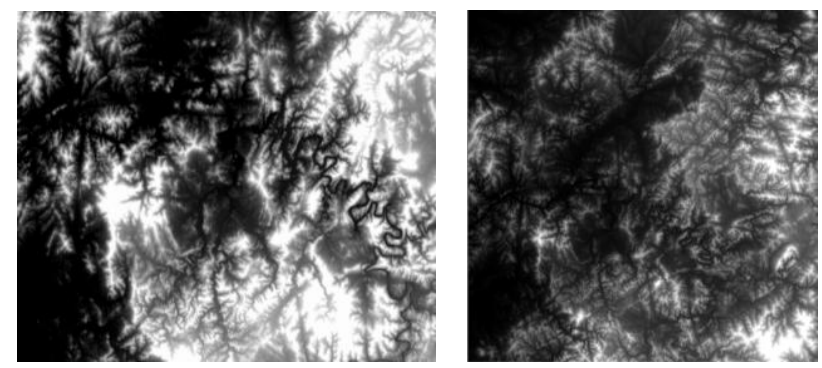

Figure 2. Two reference DEMs, one at $30 \mathrm{~m}$ grid spacing(left) and DTED at $90 \mathrm{~m}$ grid spacing(right).

\section{DEM MATCHING RESULTS}

\subsection{Automated DEM Matching}

In this section, we firstly compared the accuracy of absolute orientation from DEM matching according to the number of

Table 2. Accuracy of the absolute models from DEM matching with different number of grid points

\begin{tabular}{|c|c|c|c|c|c|c|c|c|c|}
\hline \multirow{2}{*}{ Sensor } & \multirow{2}{*}{$\begin{array}{l}\text { The No. of grid points } \\
\text { for matching }\end{array}$} & \multicolumn{3}{|c|}{ Left model errors(pixel) } & \multicolumn{3}{|c|}{ Right model errors(pixel) } & \multicolumn{2}{|c|}{ Object space errors (m) } \\
\hline & & Col & Row & All & $\mathrm{Col}$ & Row & All & Horizontal & Vertical \\
\hline \multirow{4}{*}{ SPOT-5 } & 211 (visual checking) & 0.56 & 1.08 & 1.21 & 1.27 & 1.57 & 2.02 & 3.85 & 3.97 \\
\hline & 303 (visual checking) & 0.62 & 0.93 & 1.12 & 1.36 & 1.32 & 1.89 & 3.65 & 3.76 \\
\hline & 411 (visual checking) & 0.58 & 0.90 & 1.07 & 1.28 & 1.20 & 1.75 & 3.45 & 3.82 \\
\hline & 5985 (automation) & 0.62 & 2.12 & 2.21 & 1.41 & 1.66 & 2.18 & 5.30 & 3.98 \\
\hline
\end{tabular}


grid points used for DEM matching and based on that, we further examined the feasibility of automatic DEM matching using a large quantity of grid points extracted automatically over the whole area. Table 2 shows the accuracy of absolute models from DEM matching with different number of grid points. The accuracy was assessed using true GCPs, 18 points as shown in table 1.

Firstly, the different number of selected grid points were tested by removing outliers though visual checking and it is observed that more reliable visually checked grid points lead to more accurate DEM matching results. Continuously, the potential of automated extraction of grid points was tested for DEM matching. We extracted automatically about 6,000 points at a regular grid interval. The accuracy of the absolute models from DEM matching with the automatic gird points was shown in table 2. Slight accuracy degradation was observed. Since there are limitations on manual acquisition and visual checking of many grid points for DEM matching, grid points generated from automatic process are preferred. Moreover, automatic grid points selection is a meaningful process in that it leads to automatic mapping of satellite image without ground control points.

Considering several DEM matching error sources such as resolution difference between two DEMs, respective errors on each DEM, in particular the reference DEM, we could expect that fully automated grid points including outliers may lead to accuracy degradation of DEM matching. Nevertheless, it is observed that DEM matching still stably worked to SPOT-5.

Through automatic DEM matching, we generated a DEM at grid spacing of $10 \mathrm{~m}$ based on absolute orientation using automatic grid selection. The DEM is shown in figure 3. We carried out accuracy assessment further by comparing the reference dataset. The DEM extracted from digital map on a scale of 1:5,000 were employed as reference data and it is shown in figure 4.

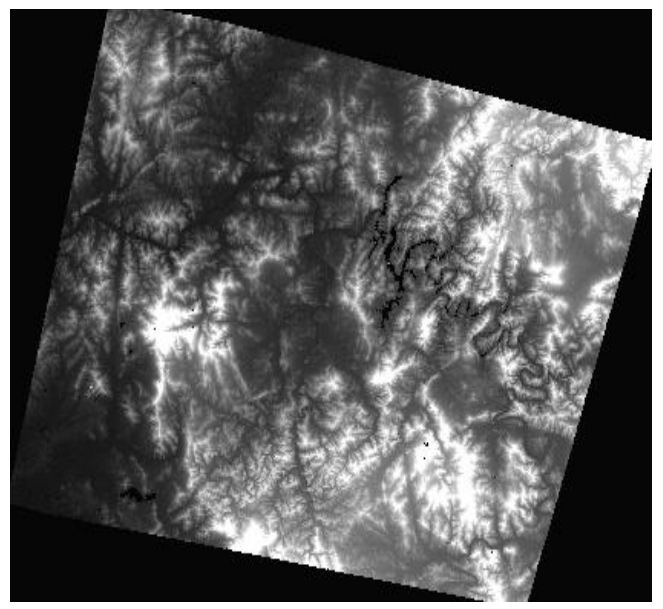

Figure 3. SPOT-5 DEM from automatic DEM Matching

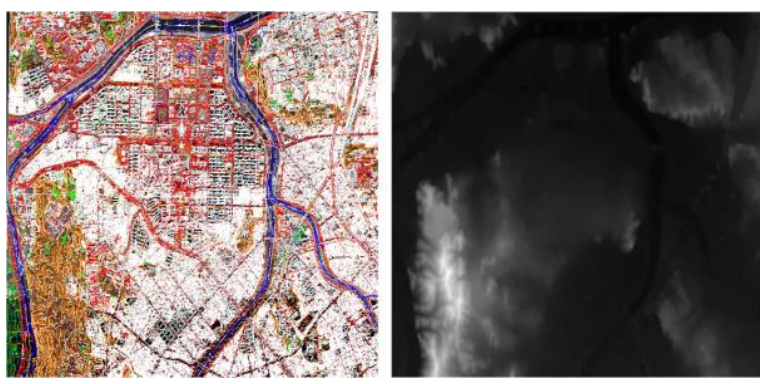

Figure 4. The reference digital map and extracted DEM

We compared about 3,000 points of the resultant DEM with corresponding points of the DEM extracted from the digital map. Height differences between the two DEMs were calculated by Mean Absolute Error(MAE) and Root Mean Square Error(RMSE). Our DEM yielded MAE of approximately $7 \mathrm{~m}$ and RMSE of approximately $12 \mathrm{~m}$. Overall, through the DEM matching experiments, we confirmed the feasibility of reliable DEM generation without ground control points.

\subsection{Applicability of the Existing 90m Global Elevation Data}

There exist several difficulties coming from differences of resolution between the relative DEM and reference DEMs such as the errors contained in the reference DEM when applying DEM matching. Considering these factors, DEM matching looks more challenging when using much lower resolution elevation dataset. For testing this feasibility, we here extended our DEM matching technique by using the existing $90 \mathrm{~m}$ global elevation data. If it is proved that the global DEMs can be sufficiently used for DEM matching, our proposed method can be used as an efficient solution to absolute orientation of high resolution satellite image without ground control points.

Using SRTM-derived DEMs(DTEDs) with gird spacing of $90 \mathrm{~m}$, we performed DEM matching with automated grid points selection and check the accuracy of absolute models. Table 3 shows the accuracy with $90 \mathrm{~m}$ elevation data and the previous $30 \mathrm{~m}$ elevation data. We could achieve the accuracy of about 3 pixel on the image space and of about $9 \mathrm{~m}$ in horizontal and $2 \mathrm{~m}$ in vertical direction on the object space. Although the issue whether this is sufficient remains, the result reflects that our DEM matching worked well for the existing elevation data at $90 \mathrm{~m}$ spacing. This is very encouraging in terms of attaining wide applicability with the existing $90 \mathrm{~m}$ resolution elevation data available worldwide.

\section{SUMMARY AND CONCLUSION}

In this paper, we exploited the DEM Matching technique further using existing global elevation dataset for absolute orientation of high resolution satellite image without ground

Table 3. Automatic DEM matching results using 90m existing global elevation data

\begin{tabular}{|c|c|c|c|c|c|c|c|c|}
\hline \multirow{2}{*}{} & \multicolumn{3}{|c|}{ Left model errors (pixel) } & \multicolumn{2}{c|}{ Right model errors (pixel) } & \multicolumn{2}{c|}{ Object space errors (m) } \\
\cline { 2 - 10 } & Col & Row & All & Col & Row & All & Horizontal & Vertical \\
\hline 30m existing elevation data & 0.62 & 2.12 & 2.21 & 1.41 & 1.66 & 2.18 & 5.30 & 3.98 \\
\hline 90m existing elevation data & 2.60 & 2.44 & 3.57 & 2.36 & 2.10 & 3.16 & 9.03 & 1.99 \\
\hline
\end{tabular}


control points. We improved our previous research for DEM matching of pushbroom images: the feasibility of automatic absolute orientation and the use of the DTED dataset at $90 \mathrm{~m}$ spacing was demonstrated.

We showed that the DEM matching could establish absolute models of high resolution satellite image without ground control points. In particular, the applicability of $90 \mathrm{~m}$ existing global elevation data is very encouraging. We look forward to contributing to understanding of DEM matching for linear pushbroom images and of solution to precise mapping without ground control points.

\section{ACKNOWLEDGEMENTS}

This work was supported in part by Basic Science Research Program through the National Research Foundation of Korea funded by the Ministry of Education, Science and Technology (2011-0009721) and by the supporting project to educate GIS experts funded by the Ministry of Land, Transport and Maritime Affairs of the Government of Korea.

\section{REFERENCES}

Ebner, H., Ohlhof, T., 1994. Utilization of Ground Control Points for image orientation without point identification in image space. International Archives of Photogrammetry and Remote Sensing 30 (Part 3/1), pp. 206-211.

Ebner, H., Strunz, G., 1988. Combined point determination using Digital Terrain Models as control information. International Archives of Photogrammetry and Remote Sensing 27 (Part B11/3), 578-587.

Ebner, H., Strunz, G., Colomina, I., 1991. Block triangulation with aerial and space imagery using DTM as control information, ACSM-ASPRS Annual Convention Technical Papers, Baltimore, March 25-29, vol. 5, pp. 76-85.

Gianinetto, M., Scaioni, M., 2008. Automated geometric correction of high resolution pushbroom satellite data, Photogrammetric Engineering \& Remote Sensing, 74(1):107116.

Jacobsen, K., 2005. High resolution satellite imaging systems overview, Photogrammetrie Fernerkundung Geoinformation (PFG) (6), 487-496.

Muller, R., Krauß, T., Lehner, M., Reinartz, P., 2007. Automatic production of a European orthoimage coverage within the GMES land fast track service using Spot 4/5 and IRS-P6 Liss III data. International Archives of Photogrammetry, Remote Sensing and Spatial Information Sciences 36 (Part I/W51), 6 p. (on CDROM).

Kim, T., Im, Y.-J., 2003. Automatic satellite image registration by combination of stereo matching and random sample consensus. IEEE Transactions on Geoscience and Remote Sensing, 41 (5):1111-1117.

Kim, T., Jeong, J., 2010. Precise mapping of high resolution satellite images without ground control points. International Archives of Photogrammetry, Remote Sensing and Spatial Information Sciences 38 (Part 1), 5, on CDROM.
Kim, T., Jeong, J., 2011. DEM Matching for bias compensation of rigorous pushbroom sensor models, ISPRS journal of photogrammetry and remote sensing, 66(5):692-699.

Reinartz P., Muller R. and Schwind P., 2011. Orthorectification of VHR optical satellite data exploiting the geometric accuracy of TerraSAR-X data, ISPRS journal of photogrammetry and remote sensing, 66(1):124-132.

Rosenholm, D., Torlegard, K., 1988. Three-dimensional absolute orientation of stereo models using digital elevation models. Photogrammetric Engineering \& Remote Sensing, 54 (10):1385-1389.

Sim D.-G., Park, R.-H., 2002. Localization based on DEM matching using multiple aerial image pairs, IEEE transactions on image processing, 11(1):52-55. 